\title{
Continuity of Barycentric Coordinates in Euclidean Topological Spaces
}

\author{
Karol Pąk \\ Institute of Informatics \\ University of Białystok \\ Poland
}

\begin{abstract}
Summary. In this paper we present selected properties of barycentric coordinates in the Euclidean topological space. We prove the topological correspondence between a subset of an affine closed space of $\mathcal{E}^{n}$ and the set of vectors created from barycentric coordinates of points of this subset.
\end{abstract}

MML identifier: RLAFFIN3, version: $7.11 .07 \quad 4.160 .1126$

The terminology and notation used here have been introduced in the following articles: [1], [3], [15], [25], [13], [18], [5], [4], [6], [12], [7], [8], [33], [21], [24], [2], [22], [20], [17], [30], [31], [23], [10], [28], [26], [11], [16], [29], [14], [19], [27], [32], and $[9]$.

\section{Preliminaries}

For simplicity, we adopt the following rules: $x$ denotes a set, $n, m, k$ denote natural numbers, $r$ denotes a real number, $V$ denotes a real linear space, $v, w$ denote vectors of $V, A_{1}$ denotes a finite subset of $V$, and $A_{2}$ denotes a finite affinely independent subset of $V$.

One can prove the following propositions:

(1) For all real-valued finite sequences $f_{1}, f_{2}$ and for every real number $r$ holds $\left(\operatorname{Intervals}\left(f_{1}, r\right)\right)^{\frown \operatorname{Intervals}}\left(f_{2}, r\right)=\operatorname{Intervals}\left(f_{1} \frown f_{2}, r\right)$.

(2) Let $f_{1}, f_{2}$ be finite sequences. Then $x \in \prod\left(f_{1} \frown f_{2}\right)$ if and only if there exist finite sequences $p_{1}, p_{2}$ such that $x=p_{1} \frown p_{2}$ and $p_{1} \in \prod f_{1}$ and $p_{2} \in \prod f_{2}$. 
(3) $V$ is finite dimensional iff $\Omega_{V}$ is finite dimensional.

Let $V$ be a finite dimensional real linear space. One can verify that every affinely independent subset of $V$ is finite.

Let us consider $n$. One can check that $\mathcal{E}_{\mathrm{T}}^{n}$ is add-continuous and multcontinuous and $\mathcal{E}_{\mathrm{T}}^{n}$ is finite dimensional.

In the sequel $p_{3}$ denotes a point of $\mathcal{E}_{\mathrm{T}}^{n}, A_{3}$ denotes a subset of $\mathcal{E}_{\mathrm{T}}^{n}, A_{4}$ denotes an affinely independent subset of $\mathcal{E}_{\mathrm{T}}^{n}$, and $A_{5}$ denotes a subset of $\mathcal{E}_{\mathrm{T}}^{k}$.

Next we state three propositions:

(4) $\operatorname{dim}\left(\mathcal{E}_{\mathrm{T}}^{n}\right)=n$

(5) Let $V$ be a finite dimensional real linear space and $A$ be an affinely independent subset of $V$. Then $\overline{\bar{A}} \leq 1+\operatorname{dim}(V)$.

(6) Let $V$ be a finite dimensional real linear space and $A$ be an affinely independent subset of $V$. Then $\overline{\bar{A}}=\operatorname{dim}(V)+1$ if and only if Affin $A=\Omega_{V}$.

\section{Open and Closed Subsets of a Subspace of the Euclidean TOPOLOGicAl SpaCE}

One can prove the following propositions:

(7) If $k \leq n$ and $A_{3}=\left\{v \in \mathcal{E}_{\mathrm{T}}^{n}: v\left\lceil k \in A_{5}\right\}\right.$, then $A_{3}$ is open iff $A_{5}$ is open.

(8) Let $A$ be a subset of $\mathcal{E}_{\mathrm{T}}^{k+n}$. Suppose $A=\{v \frown(n \mapsto 0): v$ ranges over elements of $\left.\mathcal{E}_{\mathrm{T}}^{k}\right\}$. Let $B$ be a subset of $\mathcal{E}_{\mathrm{T}}^{k+n}\lceil A$. Suppose $B=\{v ; v$ ranges over points of $\left.\mathcal{E}_{\mathrm{T}}^{k+n}: v \nmid k \in A_{5} \wedge v \in A\right\}$. Then $A_{5}$ is open if and only if $B$ is open.

(9) For every affinely independent subset $A$ of $V$ and for every subset $B$ of $V$ such that $B \subseteq A$ holds conv $A \cap$ Affin $B=\operatorname{conv} B$.

(10) Let $V$ be a non empty RLS structure, $A$ be a non empty set, $f$ be a partial function from $A$ to the carrier of $V$, and $X$ be a set. Then $(r \cdot f)^{\circ} X=r \cdot f^{\circ} X$.

(11) If $\langle\underbrace{0, \ldots, 0}_{n}\rangle \in A_{3}$, then Affin $A_{3}=\Omega_{\operatorname{Lin}\left(A_{3}\right)}$.

Let $V$ be a non empty additive loop structure, let $A$ be a finite subset of $V$, and let $v$ be an element of $V$. Note that $v+A$ is finite.

Let $V$ be a non empty RLS structure, let $A$ be a finite subset of $V$, and let us consider $r$. Observe that $r \cdot A$ is finite.

Next we state the proposition

(12) For every subset $A$ of $V$ holds $\overline{\bar{A}}=\overline{\overline{r \cdot A}}$ iff $r \neq 0$ or $A$ is trivial.

Let $V$ be a non empty RLS structure, let $f$ be a finite sequence of elements of $V$, and let us consider $r$. Note that $r \cdot f$ is finite sequence-like. 


\section{The Vector of Barycentric Coordinates}

Let $X$ be a finite set. A one-to-one finite sequence is said to be an enumeration of $X$ if:

(Def. 1) $\quad \operatorname{rng}$ it $=X$.

Let $X$ be a 1-sorted structure and let $A$ be a finite subset of $X$. We see that the enumeration of $A$ is a one-to-one finite sequence of elements of $X$.

In the sequel $E_{1}$ denotes an enumeration of $A_{2}$ and $E_{2}$ denotes an enumeration of $A_{4}$.

One can prove the following three propositions:

(13) Let $V$ be an Abelian add-associative right zeroed right complementable non empty additive loop structure, $A$ be a finite subset of $V, E$ be an enumeration of $A$, and $v$ be an element of $V$. Then $E+\overline{\bar{A}} \mapsto v$ is an enumeration of $v+A$.

(14) For every enumeration $E$ of $A_{1}$ holds $r \cdot E$ is an enumeration of $r \cdot A_{1}$ iff $r \neq 0$ or $A_{1}$ is trivial.

(15) Let $M$ be a matrix over $\mathbb{R}_{\mathrm{F}}$ of dimension $n \times m$. Suppose $\operatorname{rk}(M)=n$. Let $A$ be a finite subset of $\mathcal{E}_{\mathrm{T}}^{n}$ and $E$ be an enumeration of $A$. Then $\mathrm{Mx} 2 \operatorname{Tran} M \cdot E$ is an enumeration of $(\operatorname{Mx} 2 \operatorname{Tran} M)^{\circ} A$.

Let us consider $V, A_{1}$, let $E$ be an enumeration of $A_{1}$, and let us consider $x$. The functor $x \rightarrow E$ yielding a finite sequence of elements of $\mathbb{R}$ is defined as follows:

(Def. 2) $\quad x \rightarrow E=\left(x \rightarrow A_{1}\right) \cdot E$.

The following propositions are true:

(16) For every enumeration $E$ of $A_{1}$ holds $\operatorname{len}(x \rightarrow E)=\overline{\overline{A_{1}}}$.

(17) For every enumeration $E$ of $v+A_{2}$ such that $w \in \operatorname{Affin} A_{2}$ and $E=$ $E_{1}+\overline{\overline{A_{2}}} \mapsto v$ holds $w \rightarrow E_{1}=v+w \rightarrow E$.

(18) For every enumeration $r_{1}$ of $r \cdot A_{2}$ such that $v \in$ Affin $A_{2}$ and $r_{1}=r \cdot E_{1}$ and $r \neq 0$ holds $v \rightarrow E_{1}=r \cdot v \rightarrow r_{1}$.

(19) Let $M$ be a matrix over $\mathbb{R}_{\mathrm{F}}$ of dimension $n \times m$. Suppose $\operatorname{rk}(M)=n$. Let $M_{1}$ be an enumeration of $(\operatorname{Mx} 2 \operatorname{Tran} M)^{\circ} A_{4}$. If $M_{1}=\operatorname{Mx} 2 \operatorname{Tran} M \cdot E_{2}$, then for every $p_{3}$ such that $p_{3} \in$ Affin $A_{4}$ holds $p_{3} \rightarrow E_{2}=(\operatorname{Mx} 2 \operatorname{Tran} M)\left(p_{3}\right) \rightarrow$ $M_{1}$.

(20) Let $A$ be a subset of $V$. Suppose $A \subseteq A_{2}$ and $x \in$ Affin $A_{2}$. Then $x \in \operatorname{Affin} A$ if and only if for every set $y$ such that $y \in \operatorname{dom}\left(x \rightarrow E_{1}\right)$ and $E_{1}(y) \notin A$ holds $\left(x \rightarrow E_{1}\right)(y)=0$.

(21) For every $E_{1}$ such that $x \in \operatorname{Affin} A_{2}$ holds $x \in \operatorname{Affin}\left(E_{1}{ }^{\circ} \operatorname{Seg} k\right)$ iff $x \rightarrow$ $E_{1}=\left(\left(x \rightarrow E_{1}\right)\lceil k)^{\frown}\left(\left(\overline{\overline{A_{2}}}-{ }^{\prime} k\right) \mapsto 0\right)\right.$.

(22) For every $E_{1}$ such that $k \leq \overline{\overline{A_{2}}}$ and $x \in \operatorname{Affin} A_{2}$ holds $x \in \operatorname{Affin}\left(A_{2} \backslash\right.$ $E_{1}^{\circ}$ Seg $\left.k\right)$ iff $x \rightarrow E_{1}=(k \mapsto 0)^{\frown}\left(\left(x \rightarrow E_{1}\right)_{\lfloor k}\right)$. 
(23) Suppose $\langle\underbrace{0, \ldots, 0}_{n}\rangle \in A_{4}$ and $E_{2}\left(\operatorname{len} E_{2}\right)=\langle\underbrace{0, \ldots, 0}_{n}\rangle$. Then

(i) $\operatorname{rng}\left(E_{2} \uparrow\left(\overline{\overline{A_{4}}}-^{\prime} 1\right)\right)=A_{4} \backslash\{\langle\underbrace{0, \ldots, 0}_{n}\rangle$, and

(ii) for every subset $A$ of the $n$-dimension vector space over $\mathbb{R}_{\mathrm{F}}$ such that $A_{4}=A$ holds $E_{2} \uparrow\left(\overline{\overline{A_{4}}}-{ }^{\prime} 1\right)$ is an ordered basis of $\operatorname{Lin}(A)$.

(24) Let $A$ be a subset of the $n$-dimension vector space over $\mathbb{R}_{\mathrm{F}}$. Suppose $A_{4}=A$ and $\langle\underbrace{0, \ldots, 0}_{n}\rangle \in A_{4}$ and $E_{2}\left(\operatorname{len} E_{2}\right)=\langle\underbrace{0, \ldots, 0}_{n}\rangle$. Let $B$ be an ordered basis of $\operatorname{Lin}(A)$. If $B=E_{2} \uparrow\left(\overline{\overline{A_{4}}}-{ }^{\prime} 1\right)$, then for every element $v$ of $\operatorname{Lin}(A)$ holds $v \rightarrow B=\left(v \rightarrow E_{2}\right)\left\lceil\left(\overline{\overline{A_{4}}}-{ }^{\prime} 1\right)\right.$.

(25) For all $E_{2}, A_{3}$ such that $k \leq n$ and $\overline{\overline{A_{4}}}=n+1$ and $A_{3}=\left\{p_{3}:\left(p_{3} \rightarrow\right.\right.$ $\left.E_{2}\right)\left\lceil k \in A_{5}\right\}$ holds $A_{5}$ is open iff $A_{3}$ is open.

(26) For every $E_{2}$ such that $k \leq n$ and $\overline{\overline{A_{4}}}=n+1$ and $A_{3}=\left\{p_{3}:\left(p_{3} \rightarrow\right.\right.$ $\left.E_{2}\right)\left\lceil k \in A_{5}\right\}$ holds $A_{5}$ is closed iff $A_{3}$ is closed.

Let us consider $n$. One can verify that every subset of $\mathcal{E}_{\mathrm{T}}^{n}$ which is affine is also closed.

In the sequel $p_{4}$ denotes an element of $\mathcal{E}_{\mathrm{T}}^{n} \uparrow$ Affin $A_{4}$.

Next we state two propositions:

(27) For every $E_{2}$ and for every subset $B$ of $\mathcal{E}_{\mathrm{T}}^{n} \uparrow$ Affin $A_{4}$ such that $k<\overline{\overline{A_{4}}}$ and $B=\left\{p_{4}:\left(p_{4} \rightarrow E_{2}\right)\left\lceil k \in A_{5}\right\}\right.$ holds $A_{5}$ is open iff $B$ is open.

(28) Let given $E_{2}$ and $B$ be a subset of $\mathcal{E}_{\mathrm{T}}^{n} \uparrow$ Affin $A_{4}$. Suppose $k<\overline{\overline{A_{4}}}$ and $B=\left\{p_{4}:\left(p_{4} \rightarrow E_{2}\right)\left\lceil k \in A_{5}\right\}\right.$. Then $A_{5}$ is closed if and only if $B$ is closed.

Let us consider $n$ and let $p, q$ be points of $\mathcal{E}_{\mathrm{T}}^{n}$. Observe that halfline $(p, q)$ is closed.

\section{Continuity of Barycentric Coordinates}

Let us consider $V$, let $A$ be a subset of $V$, and let us consider $x$. The functor $\vdash(A, x)$ yielding a function from $V$ into $\mathbb{R}^{\mathbf{1}}$ is defined as follows:

(Def. 3) $\quad(\vdash(A, x))(v)=(v \rightarrow A)(x)$.

One can prove the following four propositions:

(29) For every subset $A$ of $V$ such that $x \notin A$ holds $\vdash(A, x)=\Omega_{V} \longmapsto 0$.

(30) For every affinely independent subset $A$ of $V$ such that $\vdash(A, x)=$ $\Omega_{V} \longmapsto 0$ holds $x \notin A$.

(31) $\vdash\left(A_{4}, x\right)\left\lceil\right.$ Affin $A_{4}$ is a continuous function from $\mathcal{E}_{\mathrm{T}}^{n} \uparrow$ Affin $A_{4}$ into $\mathbb{R}^{\mathbf{1}}$.

(32) If $\overline{\overline{A_{4}}}=n+1$, then $\vdash\left(A_{4}, x\right)$ is continuous.

Let us consider $n, A_{4}$. Note that conv $A_{4}$ is closed.

We now state the proposition 
(33) If $\overline{\overline{A_{4}}}=n+1$, then $\operatorname{Int} A_{4}$ is open.

\section{REFERENCES}

[1] Grzegorz Bancerek. Cardinal numbers. Formalized Mathematics, 1(2):377-382, 1990.

[2] Grzegorz Bancerek. The fundamental properties of natural numbers. Formalized Mathematics, 1(1):41-46, 1990.

[3] Grzegorz Bancerek. König's theorem. Formalized Mathematics, 1(3):589-593, 1990.

[4] Grzegorz Bancerek and Krzysztof Hryniewiecki. Segments of natural numbers and finite sequences. Formalized Mathematics, 1(1):107-114, 1990.

[5] Czesław Byliński. Binary operations applied to finite sequences. Formalized Mathematics, 1(4):643-649, 1990.

[6] Czesław Byliński. Finite sequences and tuples of elements of a non-empty sets. Formalized Mathematics, 1(3):529-536, 1990.

[7] Czesław Byliński. Functions and their basic properties. Formalized Mathematics, 1(1):5565, 1990.

[8] Czesław Byliński. Functions from a set to a set. Formalized Mathematics, 1(1):153-164, 1990.

[9] Czesław Byliński. Some basic properties of sets. Formalized Mathematics, 1(1):47-53, 1990.

[10] Czesław Byliński. Introduction to real linear topological spaces. Formalized Mathematics, 13(1):99-107, 2005.

[11] Jing-Chao Chen. The Steinitz theorem and the dimension of a real linear space. Formalized Mathematics, 6(3):411-415, 1997.

[12] Agata Darmochwał. Finite sets. Formalized Mathematics, 1(1):165-167, 1990.

[13] Agata Darmochwał. The Euclidean space. Formalized Mathematics, 2(4):599-603, 1991.

[14] Agata Darmochwał and Yatsuka Nakamura. Metric spaces as topological spaces - fundamental concepts. Formalized Mathematics, 2(4):605-608, 1991.

[15] Noboru Endou, Takashi Mitsuishi, and Yasunari Shidama. Convex sets and convex combinations. Formalized Mathematics, 11(1):53-58, 2003.

[16] Noboru Endou, Takashi Mitsuishi, and Yasunari Shidama. Dimension of real unitary space. Formalized Mathematics, 11(1):23-28, 2003.

[17] Krzysztof Hryniewiecki. Basic properties of real numbers. Formalized Mathematics, 1(1):35-40, 1990.

[18] Artur Korniłowicz. The correspondence between $n$-dimensional Euclidean space and the product of $n$ real lines. Formalized Mathematics, 18(1):81-85, 2010, doi: 10.2478/v10037010-0011-0.

[19] Eugeniusz Kusak, Wojciech Leończuk, and Michał Muzalewski. Abelian groups, fields and vector spaces. Formalized Mathematics, 1(2):335-342, 1990.

[20] Anna Lango and Grzegorz Bancerek. Product of families of groups and vector spaces. Formalized Mathematics, 3(2):235-240, 1992.

[21] Robert Milewski. Associated matrix of linear map. Formalized Mathematics, 5(3):339345, 1996.

[22] Beata Padlewska and Agata Darmochwał. Topological spaces and continuous functions. Formalized Mathematics, 1(1):223-230, 1990.

[23] Karol Pąk. Affine independence in vector spaces. Formalized Mathematics, 18(1):87-93, 2010, doi: 10.2478/v10037-010-0012-z.

[24] Karol Pąk. Linear transformations of Euclidean topological spaces. Formalized Mathematics, 19(2):103-108, 2011, doi: 10.2478/v10037-011-0016-3.

[25] Andrzej Trybulec. Domains and their Cartesian products. Formalized Mathematics, 1(1):115-122, 1990

[26] Wojciech A. Trybulec. Basis of real linear space. Formalized Mathematics, 1(5):847-850, 1990.

[27] Wojciech A. Trybulec. Basis of vector space. Formalized Mathematics, 1(5):883-885, 1990.

[28] Wojciech A. Trybulec. Vectors in real linear space. Formalized Mathematics, 1(2):291-296, 1990.

[29] Zinaida Trybulec. Properties of subsets. Formalized Mathematics, 1(1):67-71, 1990.

[30] Edmund Woronowicz. Relations and their basic properties. Formalized Mathematics, 1(1):73-83, 1990. 
[31] Edmund Woronowicz. Relations defined on sets. Formalized Mathematics, 1(1):181-186, 1990.

[32] Hiroshi Yamazaki and Yasunari Shidama. Algebra of vector functions. Formalized Mathematics, 3(2):171-175, 1992.

[33] Katarzyna Zawadzka. The sum and product of finite sequences of elements of a field. Formalized Mathematics, 3(2):205-211, 1992.

Received December 21, 2010 